\title{
Detecting transcription of ribosomal protein pseudogenes in diverse human tissues from RNA-seq data
}

Peter Tonner ${ }^{1}$, Vinodh Srinivasasainagendra ${ }^{2}$, Shaojie Zhang ${ }^{{ }^{*}}$ and Degui Zhi ${ }^{2^{*}}$

\begin{abstract}
Background: Ribosomal proteins (RPs) have about 2000 pseudogenes in the human genome. While anecdotal reports for RP pseudogene transcription exists, it is unclear to what extent these pseudogenes are transcribed. The RP pseudogene transcription is difficult to identify in microarrays due to potential cross-hybridization between transcripts from the parent genes and pseudogenes. Recently, transcriptome sequencing (RNA-seq) provides an opportunity to ascertain the transcription of pseudogenes. A challenge for pseudogene expression discovery in RNA-seq data lies in the difficulty to uniquely identify reads mapped to pseudogene regions, which are typically also similar to the parent genes.

Results: Here we developed a specialized pipeline for pseudogene transcription discovery. We first construct a "composite genome" that includes the entire human genome sequence as well as mRNA sequences of real ribosomal protein genes. We then map all sequence reads to the composite genome, and only exact matches were retained. Moreover, we restrict our analysis to strictly defined mappable regions and calculate the RPKM values as measurement of pseudogene transcription levels. We report evidences for the transcription of RP pseudogenes in 16 human tissues. By analyzing the Human Body Map 2.0 study RNA-sequencing data using our pipeline, we identified that one ribosomal protein (RP) pseudogene (PGOHUM-249508) is transcribed with RPKM 170 in thyroid. Moreover, three other RP pseudogenes are transcribed with RPKM $>10$, a level similar to that of the normal RP genes, in white blood cell, kidney, and testes, respectively. Furthermore, an additional thirteen RP pseudogenes are of RPKM > 5, corresponding to the 20-30 percentile among all genes. Unlike ribosomal protein genes that are constitutively expressed in almost all tissues, RP pseudogenes are differentially expressed, suggesting that they may contribute to tissue-specific biological processes.
\end{abstract}

Conclusions: Using a specialized bioinformatics method, we identified the transcription of ribosomal protein pseudogenes in human tissues using RNA-seq data.

Keywords: Ribosomal protein, Pseudogene, Transcription, RNA-seq data

\section{Background}

Pseudogenes are "fossil" copies of functional genes that have lost their potential as DNA templates for functional products [1-6]. While the definition of pseudogenes is still somewhat fuzzy, most of them are defined operationally by bioinformatics criteria, e.g., genomic scans of signatures of homology to known genes. Ribosomal

\footnotetext{
* Correspondence: shzhang@eecs.ucf.edu; dzhi@soph.uab.edu

${ }^{1}$ Department of Electrical Engineering and Computer Science, University of Central Florida, Orlando, FL 32816, USA

${ }^{2}$ Department of Biostatistics, Section on Statistical Genetics, University of Alabama at Birmingham, Birmingham, AL 35294, USA
}

protein (RP) pseudogenes represent the largest class of pseudogenes found in the human genome: over 2000 ribosomal protein pseudogenes are identified by bioinformatics scan of genomic sequence [5].

These pseudogenes are commonly thought to be nonfunctional due to the lack of promoters and/or the presence of loss of function mutations. Indeed, the vast majority of these pseudogenes either carry dysfunctional mutations such as in-frame stop codons, or lack of proper regulatory sequences, such as promoters, mTOP signals, and first introns [7]. Interestingly, three RP
C Biomed Central

(C) 2012 Tonner et al.; licensee BioMed Central Ltd. This is an Open Access article distributed under the terms of the Creative Commons Attribution License (http://creativecommons.org/licenses/by/2.0), which permits unrestricted use, distribution, and reproduction in any medium, provided the original work is properly cited. 
pseudogenes, with $89 \%-95 \%$ sequence identity to their parent (progenitor) RP genes, were found to be transcribed and seem to be functional, by a bioinformatics scan of cDNA and expression sequence tag (EST) databases and confirmation by PCR and Northern blot [8]. A genome-wide bioinformatics scan identified over 2000 potential pseudogenes [5]. Moreover, it was found [9] that the six RP pseudogenes shared at syntenic loci between the human and the mouse genomes are more conserved than other RP pseudogenes.

However, data were lacking to experimentally validate pseudogene expression. It is unclear from the literature whether the reported cases are merely anecdotal or that pseudogenes do play some cellular roles. This is largely hindered by the lack of methods for the identification of pseudogenes transcription. The traditional method of transcriptome profiling, gene expression microarray, is not sensitive in distinguishing transcripts among very similar gene sequences.

Recent advancements of next-generation sequencing allow for direct massive transcriptome sequencing (RNA-seq), and thus providing unprecedented insights into all transcribed sequences. For example, RNA-seq has been applied to detect complex transcriptional activities such as alternative splicing $[10,11]$ and allelicspecific expression [12]. Recently, RNA-seq has been applied to reveal RNA editing events [13]. However, to the best of our knowledge, there were yet no attempts to detect the transcription of pseudogenes in RNA-seq data. The main challenge for pseudogene identification in RNA-seq data is the difficulty of high fidelity read mapping. Because sequences of pseudogenes are highly similar to the sequences of the mRNAs of the parent genes, specialized read mapping methods are required to detect reads unambiguously generated from pseudogenes.

In this study, we conduct a bioinformatics analysis of pseudogene expression using RNA-sequencing data of 16 human tissues of the Illumina Human Body Map 2.0 project. We first describe our new computational pipeline for detecting pseudogene expression that disentangles sequencing reads of pseudogenes from those of the parent genes, with consideration of possible mismatches due to SNPs and RNA-editing. This is followed by a description of our findings and a discussion of their implications.

\section{Results}

Illumina Human Body Map 2.0 RNA-seq data

The Human Body Map 2.0 Project by Illumina generated RNA-seq data for 16 different human tissues (adipose, adrenal, brain, breast, colon, heart, kidney, liver, lung, lymph node, ovary, prostate, skeletal muscle, testes, thyroid, and white blood cells). In our analysis we used the 75 bps single read data, with one lane of HiSeq 2000

Table 1 The number of reads mapped to RefSeq sequences and RP pseudogenes for both the composite genome and the unaltered human genome (hg18) for each tissue

\begin{tabular}{|c|c|c|c|c|c|c|}
\hline \multirow[t]{2}{*}{ Tissue } & \multicolumn{3}{|c|}{ RP Pseudogenes } & \multicolumn{3}{|c|}{ RefSeq Sequences } \\
\hline & Composite genome & hg18 & Ratio & Composite genome & hg18 & Ratio \\
\hline Adipose & 708 & 22613 & 0.03 & 22018081 & 23753007 & 0.93 \\
\hline Adrenal & 2439 & 22280 & 0.11 & 19436010 & 21074345 & 0.92 \\
\hline Brain & 712 & 3853 & 0.18 & 11585759 & 12357788 & 0.94 \\
\hline Breast & 1603 & 15951 & 0.10 & 22962845 & 23060856 & 1.00 \\
\hline Colon & 1066 & 22948 & 0.05 & 21458813 & 21618780 & 0.99 \\
\hline Heart & 562 & 2374 & 0.24 & 12622175 & 13502482 & 0.93 \\
\hline Kidney & 1341 & 5928 & 0.23 & 20630500 & 22278600 & 0.93 \\
\hline Liver & 328 & 2359 & 0.14 & 16689110 & 17958332 & 0.93 \\
\hline Lung & 1128 & 11918 & 0.09 & 28648503 & 31274037 & 0.92 \\
\hline Lymph & 1456 & 14010 & 0.10 & 22126747 & 23998836 & 0.92 \\
\hline Muscle & 459 & 8705 & 0.05 & 25836676 & 27662752 & 0.93 \\
\hline Ovary & 1501 & 34608 & 0.04 & 21842763 & 23663484 & 0.92 \\
\hline Prostate & 1280 & 14976 & 0.09 & 30329651 & 32822472 & 0.92 \\
\hline Testes & 1885 & 19417 & 0.10 & 23356973 & 25123824 & 0.93 \\
\hline Thyroid & 4064 & 22250 & 0.18 & 24800037 & 26627946 & 0.93 \\
\hline White Blood Cells & 1095 & 14256 & 0.08 & 25487259 & 27812790 & 0.92 \\
\hline Average & 1351 & 14902 & 0.11 & 21864493 & 23411895 & 0.94 \\
\hline
\end{tabular}

The ratio is calculated by the number of mapped reads from the composite genome over the number of mapped reads from the unaltered human genome (hg18). 
data per tissue. Standard mRNA-seq library preps were used to extract poly-A selected mRNAs.

\section{Discovery of pseudogene transcription in RNA-seq data}

Our primary goal is to detect transcriptional activities of any of the 1709 processed RP pseudogenes. In addition, we also want to provide a preliminary quantification of their level of transcription.

We developed a novel bioinformatics approach for detecting the subtle signals of pseudogene expression. Briefly, we first compiled a "composite genome" consisting of known RP gene spliced mRNA sequences and the human genome (hg18) [14]. We then mapped RNA-seq reads to the composite genome using Bowtie [15], allowing no mismatches and discarding reads mapped to more than one locus. Thus we ensured that the reads mapped to RP pseudogenes are neither from repetitive regions nor from normal RP genes. On average $89 \%$ of the reads aligning to RP pseudogenes can also be mapped to real RP spliced mRNA sequences and are removed when mapped to the composite genome (see Table 1). Furthermore, to remove mapped reads that may be caused by SNPs and RNAediting, we extended the concept of the mappability (the mappable regions of human genome is called the uniqueome) [16] and masked regions in RP pseudogenes that are duplicated in the composite genome within 4 mismatches over the $75 \mathrm{bps}$ read length. The number of reads we removed from non-unique locations in both the composite genome and hg18 genome can be seen in Table 2 . The mappability regions only correspond to the unaltered human genome locations, so all reads mapped to RP gene mRNA sequences in the composite genome are removed during this step. Additionally, the composite genome alignment lacks the reads that mapped both to the unaltered human genome locations and spliced RP gene mRNA sequences as we only retained reads aligning to a single location. With both of these groups of reads removed, the number of reads mapped uniquely in the composite genome is always less than that in the unaltered human genome. Finally, we calculated the transcription levels, as measured by the Reads Per Kilobase per Million reads (RPKM) [11] of all pseudogenes according to the mapped reads in their mappable regions. As a benchmark for normal expression levels, we also aligned reads to an unaltered genome using TopHat and measured FPKM of all RefSeq genes using Cufflinks [17]. The alignment information of reads to the composite genome, and to the unaltered genome (hg18), can be seen in Table 2. Please see Methods for details.

\section{Prevalent transcription of RP pseudogenes}

The expression levels of the top seventeen highly expressed ribosomal protein pseudogenes in 16 human tissues are shown in Figure 1 and Table 3 (See Table S1

Table 2 Statistics for each tissue sample

\begin{tabular}{|c|c|c|c|c|c|}
\hline Tissue & $\begin{array}{l}\text { Number of } \\
\text { reads in the } \\
\text { sample }\end{array}$ & $\begin{array}{l}\text { Number of } \\
\text { reads mapped } \\
\text { to the } \\
\text { composite } \\
\text { genome }\end{array}$ & $\begin{array}{l}\text { Number of } \\
\text { reads } \\
\text { mapped to } \\
\text { hg18 }\end{array}$ & $\begin{array}{l}\text { Number of reads } \\
\text { mapped uniquely } \\
\text { to the composite } \\
\text { genome }\end{array}$ & $\begin{array}{l}\text { Number of } \\
\text { reads mapped } \\
\text { uniquely to the } \\
\text { hg18 }\end{array}$ \\
\hline Adipose & $76,269,225$ & $39,499,413$ & $39,759,404$ & $27,507,408$ & $30,635,265$ \\
\hline Adrenal & $76,171,569$ & $39,330,423$ & $39,681,721$ & $29,670,249$ & $32,603,673$ \\
\hline Brain & $64,313,204$ & $21,022,913$ & $21,073,103$ & $15,785,362$ & $17,187,772$ \\
\hline Breast & $77,195,260$ & $39,355,808$ & $39,568,435$ & $30,955,674$ & $31,072,723$ \\
\hline Colon & $80,257,757$ & $39,406,195$ & $39,735,900$ & $28,393,105$ & $28,582,515$ \\
\hline Heart & $76,766,862$ & $29,030,896$ & $29,065,063$ & $17,099,881$ & $19,366,541$ \\
\hline Kidney & $79,772,393$ & $41,368,095$ & $41,488,175$ & $27,316,562$ & $30,570,744$ \\
\hline Liver & $77,453,877$ & $26,692,219$ & $26,741,073$ & $18,897,673$ & $20,715,729$ \\
\hline Lung & $81,255,438$ & $45,996,752$ & $46,211,355$ & $34,401,958$ & $37,862,617$ \\
\hline Lymph & $81,916,460$ & $41,826,888$ & $42,072,585$ & $30,451,248$ & $33,277,288$ \\
\hline Muscle & $82,864,636$ & $46,580,440$ & $46,725,392$ & $30,704,302$ & $34,165,828$ \\
\hline Ovary & $81,003,052$ & $36,922,138$ & $37,385,453$ & $28,184,877$ & $30,861,571$ \\
\hline Prostate & $83,319,902$ & $47,601,661$ & $47,965,443$ & $36,138,822$ & $39,533,973$ \\
\hline Testes & $82,044,319$ & $38,852,709$ & $39,069,136$ & $29,115,004$ & $31,927,737$ \\
\hline Thyroid & $80,246,657$ & $40,546,781$ & $40,785,090$ & $31,137,501$ & $33,939,339$ \\
\hline White Blood Cells & $82,785,673$ & $38,860,771$ & $39,098,752$ & $28,796,204$ & $31,784,122$ \\
\hline
\end{tabular}

The number of reads mapped to the composite genome (which includes spliced ribosomal protein gene sequences) and to the unaltered human genome (hy18), and the number of reads overlapped with uniqueome ("mapped uniquely") for both are shown. For the composite genome, the number of reads aligning to the entire composite genome and the unaltered hg18 human genome are shown. 


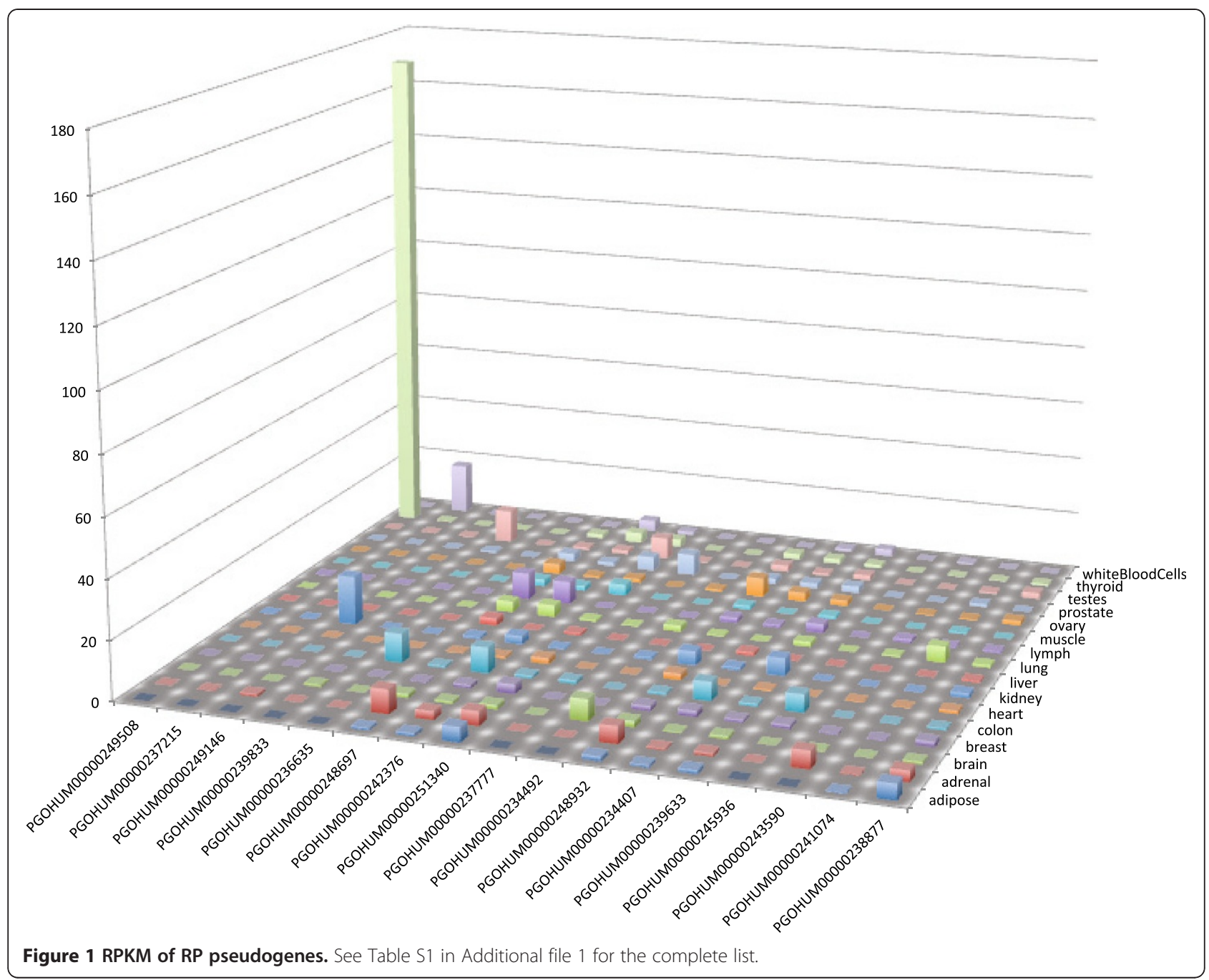

in Additional file 1 for complete list for all RP pseudogenes). As expected the majority of pseudogenes have no reads aligning to their sequence. Interestingly, there were some pseudogenes with high expression levels. One RP pseudogene (PGOHUM-249508) is transcribed with RPKM 170 in thyroid. Moreover, three additional $R P$ pseudogenes are transcribed with $R P K M>10$. Furthermore, thirteen more RP pseudogenes are of RPKM $>5$. We describe pseudogenes with an RPKM $>10$ as "highly expressed", with the understanding that they may be only representing the top 10-15 percentile of all 37,681 RefSeq genes in the Human Body Map 2.0 data set, while $R P K M>5$ represents the top 20-30 percentile (see Table 4). Below we discuss these cases in detail.

PGOHUM-249508, an RPL21 pseudogene, is expressed with $R P K M=170$ in thyroid (Figure 2). This highest expressed RP pseudogene is located in an intron of the Thyroglobulin (TG) gene. The TG gene is highly and specifically expressed in the same tissue, thyroid, and the gene encodes a glycoprotein that acts as a substrate for the synthesis of thyroxine and triiodothyronine as well as the storage of the inactive forms of thyroid hormone and iodine [18]. The transcription of this pseudogene goes beyond the annotated pseudogene region, but is less than the entire intron region. Although the pseudogene is specifically expressed in the same tissue as TG, the RP coding frame is on the reverse strand of the TG gene. Therefore, it is possible that this pseudogene is on a distinct transcript other than the TG gene. Moreover, according to UCSC genome browser [19], this pseudogene region is only conserved within the primates (between human and the Rhesus monkey), but not in other mammalian and vertebrate lineages. As a side note, the genome browser shows a peculiar conservation pattern between human and the stickleback fish, but it is likely to be an artifact of matching human genomic sequence with the RPL21 gene of stickleback fish.

Three additional pseudogenes are highly transcribed (RPKM > 10). PGOHUM-237215, an RPL7A pseudogene, is expressed $\mathrm{RPKM}=17$ in white blood cells. This 
Table 3 RP pseudogenes expression identified in Human Body Map 2.0 RNA-seq data

\begin{tabular}{|c|c|c|c|c|c|c|c|}
\hline pg-id & Parent gene & Location & Tissue with Max RPKM & Max RPKM & Total RPKM & Tissue specificity & Reads coverage \\
\hline 249508 & RPL21 & chr8:134084035-134084502 & Thyroid & 170.3 & 170.6 & 0.977 & 0.976 \\
\hline 237215 & RPL7A & chr17:6984988-6985635 & White Blood Cells & 17.3 & 18.0 & 0.881 & 0.913 \\
\hline 249146 & RPS24 & chr16:55497947-55498335 & Kidney & 16.5 & 17.6 & 0.855 & 0.693 \\
\hline 239833 & RPS11 & chr12:63076580-63077044 & Testes & 11.3 & 12.5 & 0.813 & 0.970 \\
\hline 236635 & RPL24 & chr9:72021934-72022269 & Colon & 9.7 & 9.7 & 1.000 & 0.358 \\
\hline 248697 & RPL26 & chr16:1953719-1954097 & Lymph & 9.0 & 37.9 & 0.376 & 0.854 \\
\hline 242376 & RPS20 & chr11:77202016-77202370 & Colon & 8.6 & 41.6 & 0.355 & 1.00 \\
\hline 251340 & RPLP1 & chr5:151125656-151125993 & Testes & 7.4 & 40.1 & 0.339 & 0.567 \\
\hline 237777 & RPL7 & chr3:133445060-133445787 & Prostate & 7.3 & 8.0 & 0.819 & 0.371 \\
\hline 234492 & RPL10 & chr19:9791817-9792131 & Brain & 7.2 & 10.8 & 0.635 & 0.121 \\
\hline 248932 & RPL21 & chr16:72904438-72904782 & Ovary & 6.5 & 34.3 & 0.343 & 1.00 \\
\hline 234407 & RPL39 & chr19:58143205-58143330 & Colon & 6.3 & 21.3 & 0.414 & 1.00 \\
\hline 239633 & RPL13 & chr12:6863424-6864038 & Kidney & 6.0 & 27.0 & 0.365 & 0.923 \\
\hline 245936 & RPL6 & chr4:66121772-66122638 & Colon & 5.9 & 7.2 & 0.746 & 0.109 \\
\hline 243590 & RPL32 & chr6:33155206-33155612 & Adrenal & 5.8 & 9.2 & 0.617 & 1.00 \\
\hline 241074 & RPL13A & chr2:203093361-203093957 & Lung & 5.4 & 9.7 & 0.571 & 0.995 \\
\hline 238877 & RPL11 & chr10:89695235-89695766 & Adipose & 5.2 & 20.4 & 0.387 & 0.847 \\
\hline
\end{tabular}

Expression levels of pseudogenes with their pseudogene ID (pg-id, prefix 'PGOHUM00000' omitted) are measured in terms of RPKM. Only pseudogenes with maximum RPKM $>5$ are shown. Tissue specificities are measured by the JS divergence [20]. Read coverage is the ratio of pseudogene exon length covered by uniquely mapped reads to the total pseudogene exon length.

pseudogene is located in an intergenic region. Also, the transcription unit seems to span a longer region (Figure 3). It is transcribed in a white blood cell specific fashion. PGOHUM-249146, an RPS24 pseudogene, is expressed in kidney. This pseudogene is located in the intronic region of gene SLC12A3 (Figure 4). This gene encodes a renal thiazide-sensitive sodium-chloride cotransporter that is important for electrolyte homeostasis. PGOHUM-239833, an RPS11 pseudogene, is expressed in testes. This pseudogene is located in an intergenic region (Figure 5). The comparison of read coverage with or without uniqueome filtering for these four RP pseudogenes can be been in Figures S1-S4 in Additional file 2.

Table 4 Table of FPKM expression values of RefSeq genes in 16 human tissues

\begin{tabular}{|c|c|c|c|c|c|c|c|c|c|}
\hline \multirow[t]{2}{*}{ Tissue } & \multicolumn{4}{|c|}{ FPKM } & \multicolumn{5}{|c|}{ Percentile } \\
\hline & Mean & Max & Min & std dev & $\%>1$ & $\%>2$ & $\%>5$ & $\%>10$ & $\%>15$ \\
\hline Adipose & 10.03 & 17437.80 & 0 & 143.53 & 41.07 & 32.42 & 20.62 & 12.64 & 8.96 \\
\hline Colon & 10.42 & 17376.90 & 0 & 167.07 & 40.80 & 31.61 & 19.67 & 11.93 & 8.37 \\
\hline Heart & 7.55 & 17376.90 & 0 & 122.87 & 36.61 & 27.71 & 16.09 & 9.41 & 6.43 \\
\hline Kidney & 9.97 & 16400.20 & 0 & 156.94 & 45.28 & 35.93 & 22.62 & 13.32 & 9.23 \\
\hline Liver & 14.91 & 38505.10 & 0 & 360.25 & 33.57 & 25.10 & 14.90 & 8.93 & 6.48 \\
\hline Lung & 16.29 & 57096.60 & 0 & 456.70 & 44.59 & 35.08 & 22.05 & 13.61 & 9.72 \\
\hline Lymph & 13.41 & 40919.80 & 0 & 337.50 & 45.77 & 36.77 & 23.25 & 13.70 & 9.22 \\
\hline Muscle & 8.80 & 16317.70 & 0 & 123.85 & 33.27 & 25.97 & 16.38 & 10.39 & 7.55 \\
\hline Ovary & 13.62 & 61099.30 & 0 & 359.00 & 46.27 & 37.60 & 24.59 & 15.05 & 10.37 \\
\hline Prostate & 13.72 & 39039.90 & 0 & 300.91 & 47.82 & 38.83 & 25.36 & 15.68 & 10.79 \\
\hline Testes & 12.76 & 57096.60 & 0 & 325.41 & 53.34 & 43.44 & 28.69 & 17.85 & 12.46 \\
\hline Thyroid & 12.06 & 29030.10 & 0 & 234.59 & 46.04 & 37.25 & 24.24 & 15.12 & 10.74 \\
\hline White Blood Cells & 15.42 & 40919.80 & 0 & 366.35 & 39.25 & 32.56 & 22.65 & 14.87 & 10.83 \\
\hline All Tissues & 12.23 & 61099.30 & 0 & 286.98 & 42.59 & 33.87 & 21.62 & 13.27 & 9.32 \\
\hline
\end{tabular}

Percentile columns represent the percentage of RefSeq genes in all tissues with FPKM above a given value. 


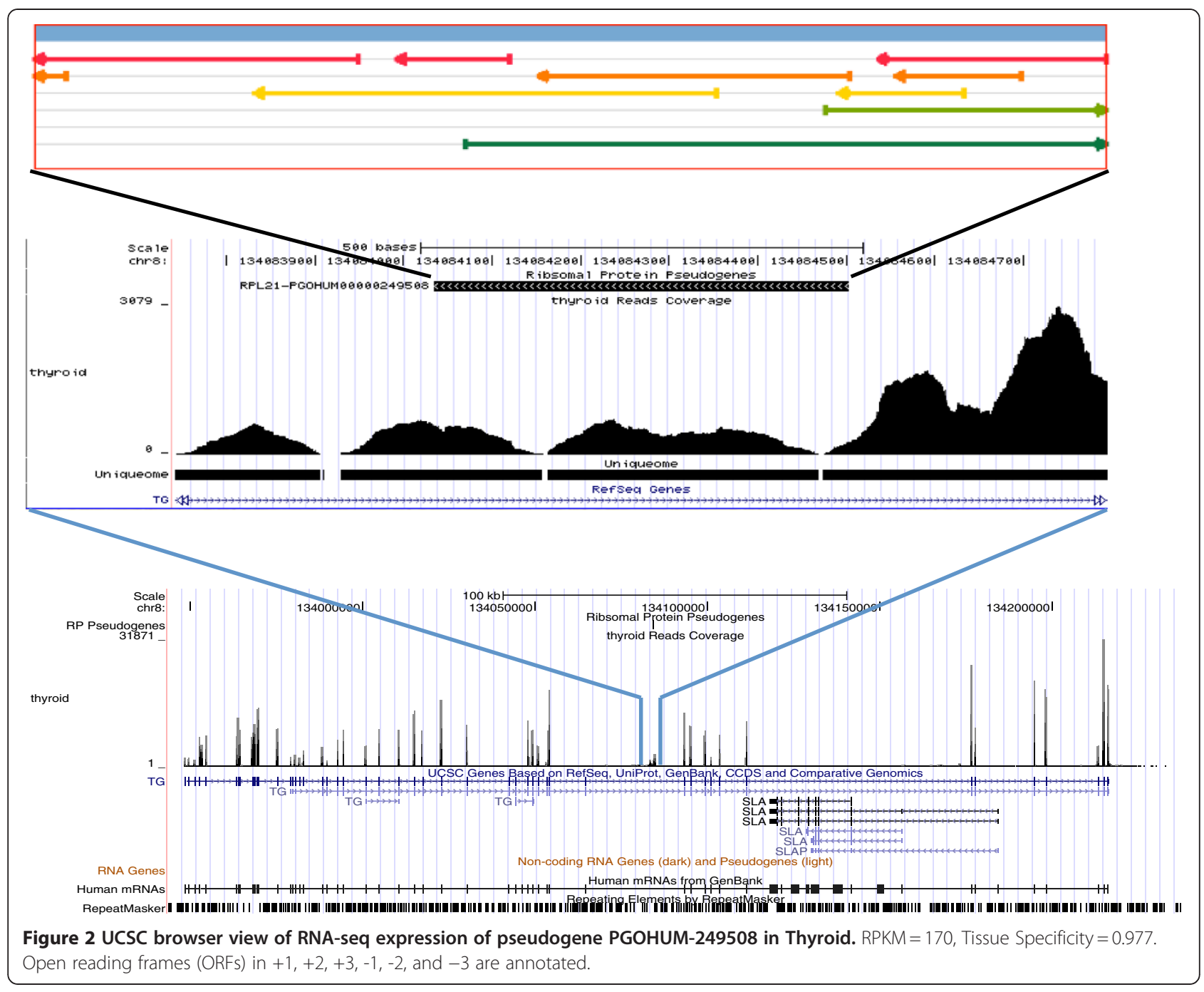

\section{Tissue-specificity of pseudogene transcription}

Many genes are expressed in a tissue-specific fashion. The Human Body Map 2.0 data allow us to study the tissue-specificity of transcriptions of these pseudogenes. We adopt the entropy-based Jensen-Shannon (JS) divergence measure used in [20]. The distributions of tissuespecificity JS divergences of RP pseudogenes versus RP genes are shown in Figure 6. In the Human Body Map 2.0 data set, all RP genes are not transcribed in a tissue specific fashion (JS divergence $<0.5$ for all RP genes) (Table S2 in Additional file 3). Unlike ribosomal protein genes that are constitutively expressed in almost all tissues, many RP pseudogenes are differentially expressed (Table S1 in Additional file 1). Among the seventeen pseudogenes with RPKM $>5$ at some tissue, 8 of them have a JS divergence $>0.5$. In fact, all of the top 4 pseudogenes with RPKM $>10$ are transcribed in a highly tissue specific fashion (JS divergence $>0.8$ ). These results suggest that these highly expressed RP pseudogenes may contribute to tissue-specific biological processes.

\section{Discussion and conclusions}

In this work, we conducted a bioinformatics analysis of the pseudogenes of ribosomal protein genes in diverse human tissues. Using our specialized pipeline, we identified several cases of pseudogene expression. Most notably, one pseudogene in an intron of the TG gene is extremely highly expressed in thyroid. Moreover, several other pseudogenes are also highly expressed. We found that many pseudogenes are expressed in a tissue-specific fashion. Also, the expression of pseudogenes seems to often go beyond the boundaries of the annotated pseudogenes. Apparently, further experimental investigations will be needed to reveal the biological relevance of these expressions.

Transcriptome sequencing, RNA-seq, provides an unprecedented opportunity to uncover many complexities of cellular gene expression. A key computational challenge in RNA-seq data analysis is to discern reads among multiple potential sources with similar sequences. In this work we focused on the detection of evidences of 


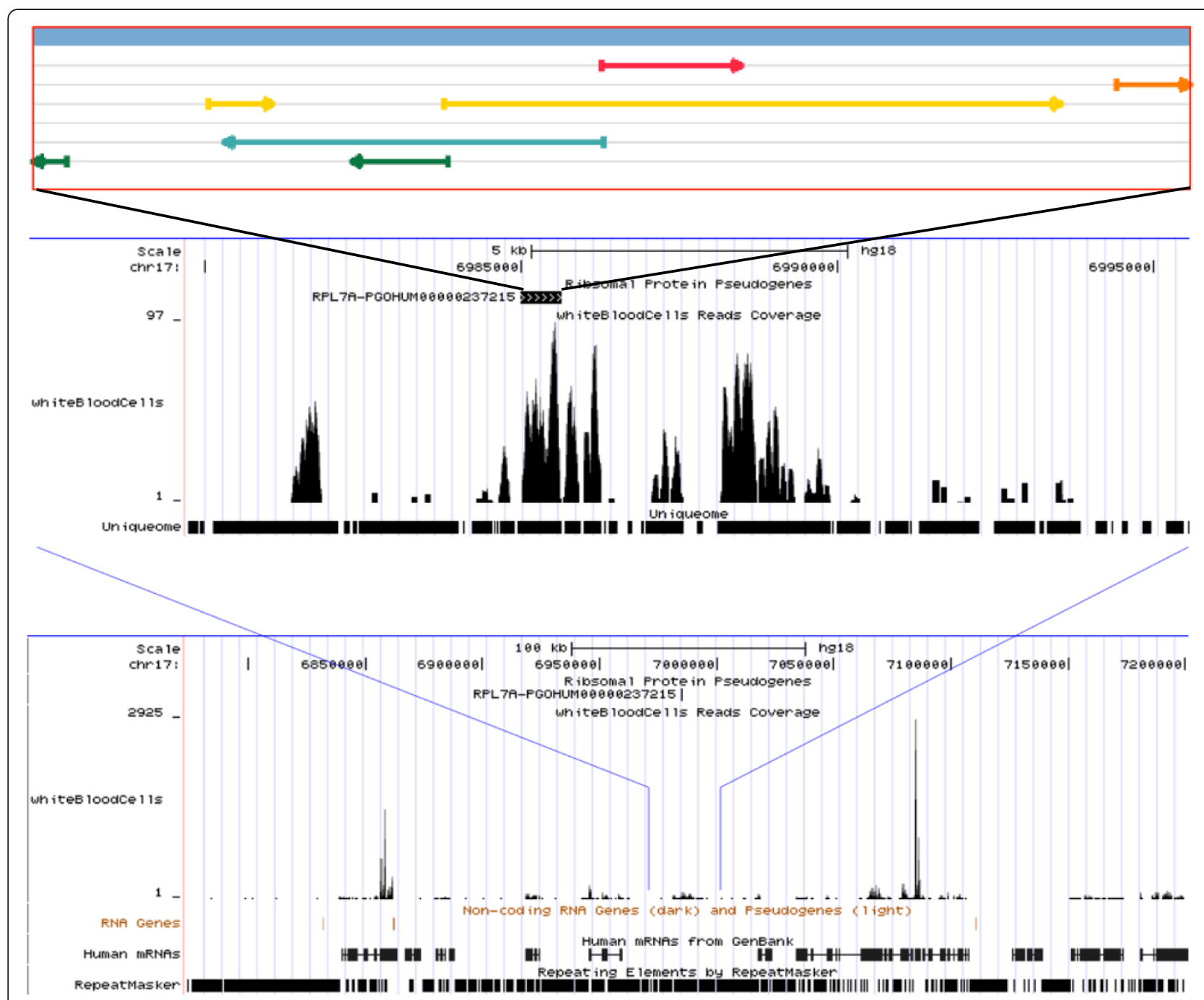

Figure 3 UCSC browser view RNA-seq expression of pseudogene PGOHUM-237215 in white blood cells. RPKM=17, Tissue Specificity $=0.881$. Open reading frames (ORFs) in $+1,+2,+3,-1,-2$, and -3 are annotated.

pseudogene expression. We used extremely strict read mapping criteria to minimize potential false positives. Admittedly we did not utilize all potential reads, especially at regions with low uniqueness. Further research may consider using looser mapping criteria combined with sophisticated statistical algorithms to take into account the mapping ambiguity.

The bioinformatics methods presented here may find application in other RNA-seq studies dealing with high similarity in reference sequences. In particular, the same methodology may be able to identify differential expression between other homologous genome regions. Studies in other fields, such as metagenomics, dealing with high similarity DNA sequences may also find benefits from strict alignment and intersection with uniquely mappable locations.

\section{Methods}

Human tissue samples

The Human Body Map 2.0 RNA-seq data for 16 human tissue samples were provided by Gary Schroth at Illumina and can be accessible from ArrayExpress (accession no. EMTAB-513). Reads were 75 base pairs long and came from the following samples: adipose, adrenal, brain, breast, colon, heart, kidney, liver, lung, lymph, muscle, ovary, prostate, testes, thyroid, and white blood cells. The samples were prepared using the Illumina mRNA-seq kit. They were made with a random priming process and are not stranded.

\section{Software and datasets}

Bowtie version 0.12.7 [15] and TopHat version 1.2.0 [21] were used for the mapping. Cufflinks version 1.0.3 [17] was used for differential expression calculation for 


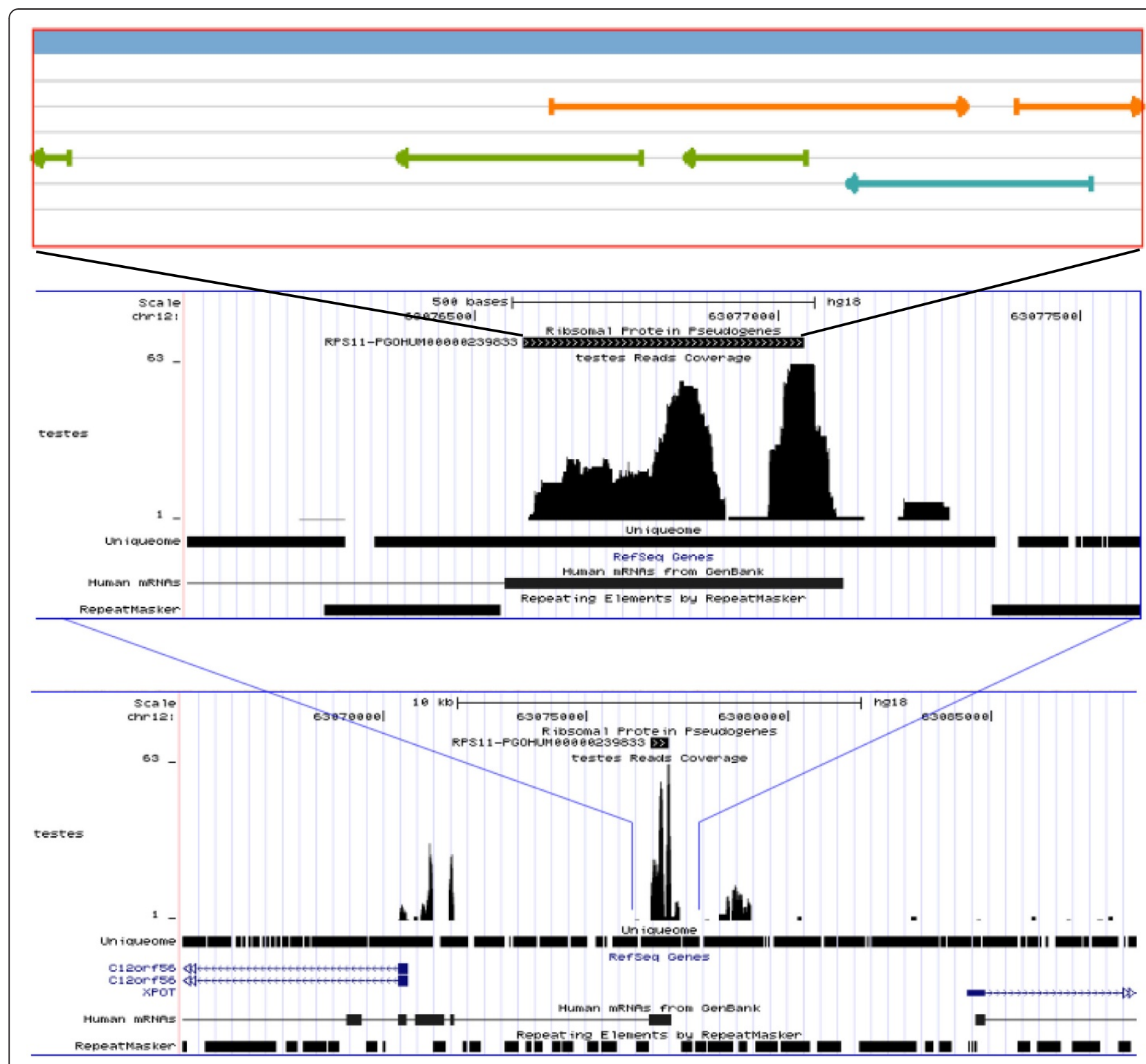

Figure 4 UCSC browser view RNA-seq expression of pseudogene PGOHUM-249146 in kidney. RPKM =16, Tissue Specificity $=0.855$. Open reading frames (ORFs) in $+1,+2,+3,-1,-2$, and -3 are annotated.

RefSeq genes. BEDTools version 2.12.0 [22] was used to analyze alignments. The uniqueome dataset was collected from the Uniqueome supplementary database [16] for human genome (hg18, NCBI Build 36.1) marking genome locations where reads of length 75 bps are unique within 4 mismatches (hg18_uniqueome. unique_starts.base-space.75.4.positive.BED). The 75 bps read length matches the RNA-seq data provided by Illumina. RefSeq genes and DNA sequences of spliced ribosomal protein genes were collected from NCBI (RefSeq database D32-6) [14]. Pseudogene annotations and sequences were downloaded from pseudogene.org [23] database (human pseudogenes build 58). Pseudogenes whose parent genes are ribosomal protein genes were selected, totaling 1788. Among them, 79 were annotated 'Duplicated'. As we are only interested in processed pseudogenes, our analysis focuses on the remaining 1709 pseudogenes. The human genome sequence (hg18) was collected from NCBI build 36.1.

\section{Composite genome}

A composite genome index was constructed with Bowtiebuild using the sequences of the human genome (hg18, NCBI build 36.1) and NCBI spliced RP gene sequences.

\section{Alignment}

RNA-seq data for each tissue was aligned using two distinct methodologies - one for pseudogenes and one for real 


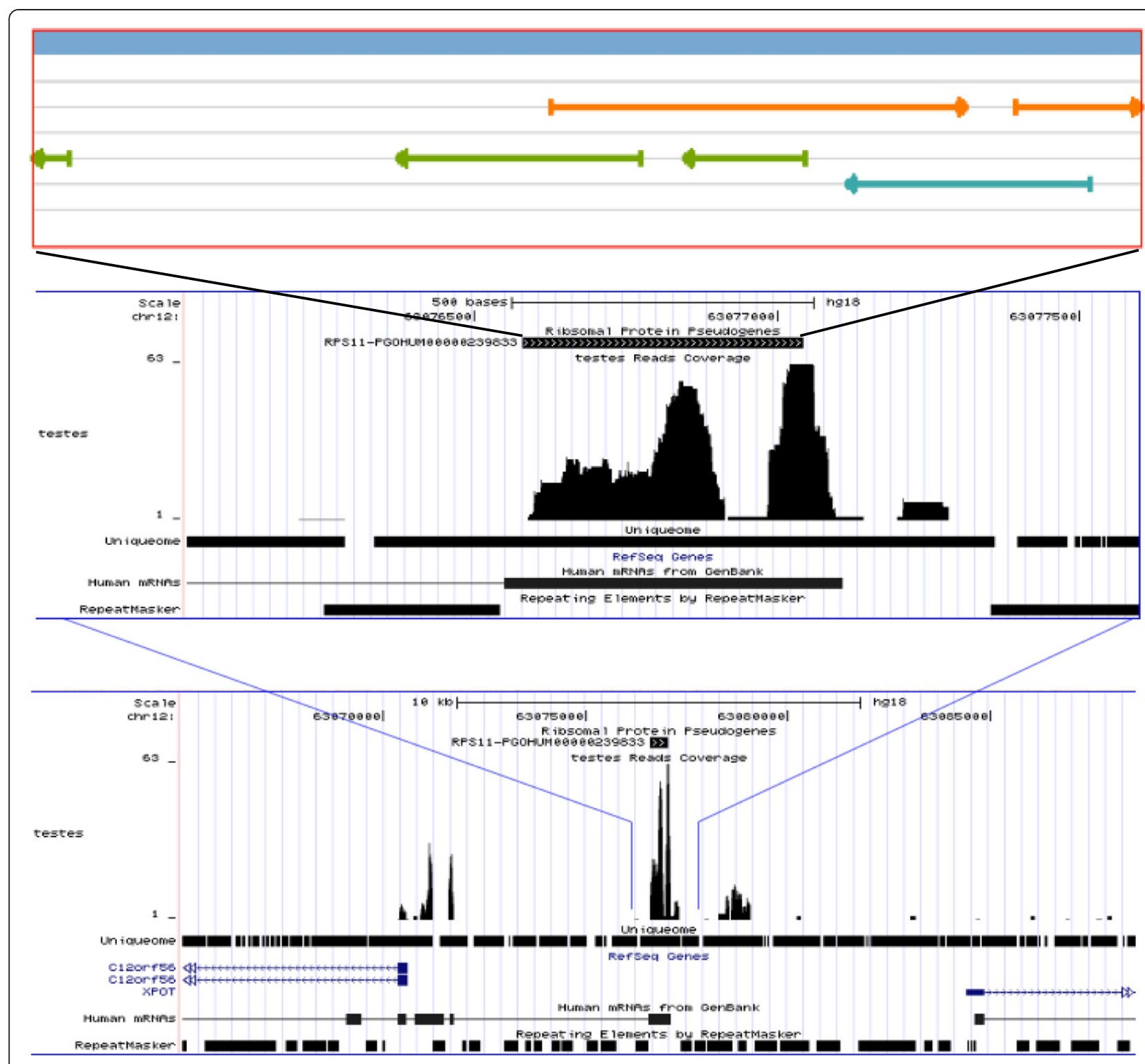

Figure 5 UCSC browser view RNA-seq expression of pseudogene PGOHUM-239833 in testes. RPKM =11, Tissue Specificity $=0.813$. Open reading frames (ORFs) in $+1,+2,+3,-1,-2$, and -3 are annotated.

genes. Pseudogene alignment protocol consists of strict alignment (Bowtie, no mismatches, report reads with only one alignment location only) to the composite genome. Real gene alignment protocol consists of strict alignment (Bowtie, no mismatches, single alignment location) to the human genome (hg18, NCBI Build 36.1).

\section{Uniqueome}

A uniqueome data set [16] was obtained for Build 36.1 marking genome locations where reads of length 75 bps are unique within 4 mismatches. Alignments for all tissues for both real genes and pseudogenes were intersected with the uniqueome dataset for all genome locations (intersectBed from BEDTools [22]). The total number of remaining reads in each alignment was counted. The uniqueome dataset was used to filter out ambiguously mapped reads.

\section{Comparative expression analysis}

Gene expression values were calculated as reads aligned to gene per kilobase of exon per million mapped reads (RPKM) [11]. The number of reads aligned to all gene exons and additionally aligning in unique locations was counted for each gene. Exon length for genes was calculated as the sum of unique positions as marked by the uniqueome across all gene exons. It is worth noting that RP pseudogenes appear spliced in the human genome and therefore have only 


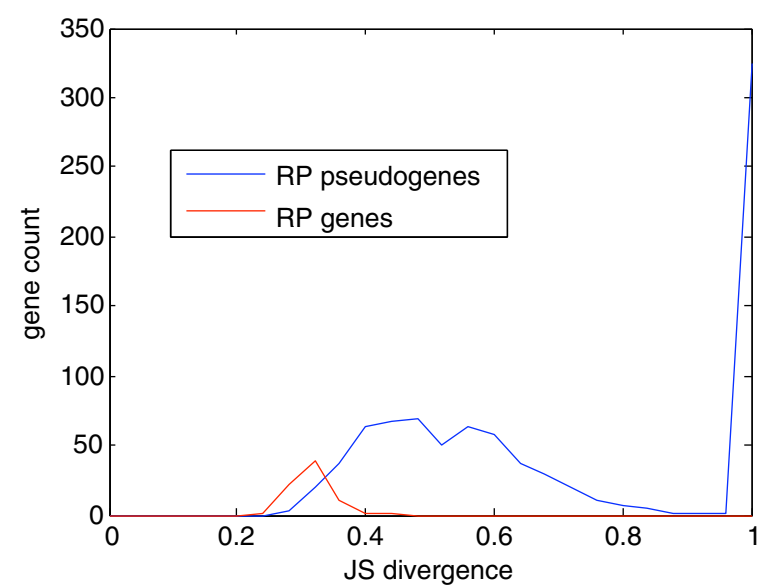

Figure 6 Distribution of tissue specificity, as measured by the JS divergence [20].

a single exon for counting aligned reads and calculating exon length.

Expression percentiles of RefSeq genes were calculated using TopHat to map reads to the human genome (hg18, NCBI build 36.1) and Cufflinks was used to calculate FPKM values of all 37,681 RefSeq genes. Expression percentiles were calculated for specific tissues and for all datasets combined.

Gene reads coverage was calculated using the coverageBed program in the BEDTools software suite. Coverage represents the fraction of RP pseudogene exon covered by reads that aligned to unique genome regions.

\section{Tissue-specificity analysis}

We followed the definition of Jensen-Shannon divergence in [20]. To avoid zero probabilities, all RPKM numbers are added by $10^{-10}$.

\section{Additional files}

Additional file 1: Table S1. RPKM expression values of RP pseudogenes in all 16 tissues.

Additional file 2: Figure S1-S4. Comparison of read coverage with or without uniqueome filtering for four RP pseudogenes.

Additional file 3: Table S2. RPKM expression values of RP genes in all 16 tissues.

\section{Competing interests}

The authors declare that they have no competing interests.

\section{Authors' contributions}

PT and VS carried out the bioinformatics analyses. PT, SZ, and DZ drafted the manuscript. SZ and DZ designed the composite genome method. DZ conceived of the study. All authors read and approved the final manuscript.

\section{Acknowledgements}

We are grateful for Gary Schroth and Illumina for the early sharing of their Human Body Map 2.0 RNA-seq data. This work is partly supported by a UAB NORC pilot grant funded by NIH grant 5P30DK056336.
Received: 12 April 2012 Accepted: 10 August 2012

Published: 21 August 2012

\section{References}

1. Balakirev ES, Ayala FJ: Pseudogenes: are they "junk" or functional DNA? Annu Rev Genet 2003, 37:123-151.

2. Harrison PM, Hegyi H, Balasubramanian S, Luscombe NM, Bertone P, Echols $\mathrm{N}$, Johnson T, Gerstein M: Molecular fossils in the human genome: identification and analysis of the pseudogenes in chromosomes 21 and 22. Genome Res 2002, 12(2):272-280,

3. Mighell AJ, Smith NR, Robinson PA, Markham AF: Vertebrate pseudogenes. FEBS Lett 2000, 468(2-3):109-114.

4. Vanin EF: Processed pseudogenes: characteristics and evolution. Annu Rev Genet 1985, 19:253-272.

5. Zhang Z, Harrison PM, Liu Y, Gerstein M: Millions of years of evolution preserved: a comprehensive catalog of the processed pseudogenes in the human genome. Genome Res 2003, 13(12):2541-2558.

6. Gerstein M, Zheng D: The real life of pseudogenes. Sci Am 2006, 295(2):48-55.

7. Chung S, Perry RP: Importance of introns for expression of mouse ribosomal protein gene rpL32. Mol Cell Biol 1989, 9(5):2075-2082.

8. Uechi T, Maeda N, Tanaka T, Kenmochi N: Functional second genes generated by retrotransposition of the X-linked ribosomal protein genes. Nucleic Acids Res 2002, 30(24):5369-5375.

9. Balasubramanian S, Zheng D, Liu YJ, Fang G, Frankish A, Carriero N, Robilotto R, Cayting P, Gerstein M: Comparative analysis of processed ribosomal protein pseudogenes in four mammalian genomes. Genome Biol 2009, 10(1):R2.

10. Sultan M, Schulz MH, Richard H, Magen A, Klingenhoff A, Scherf M, Seifert M, Borodina T, Soldatov A, Parkhomchuk D, et al: A global view of gene activity and alternative splicing by deep sequencing of the human transcriptome. Science 2008, 321(5891):956-960.

11. Mortazavi A, Williams BA, McCue K, Schaeffer L, Wold B: Mapping and quantifying mammalian transcriptomes by RNA-Seq. Nat Methods 2008, 5(7):621-628.

12. Pastinen T: Genome-wide allele-specific analysis: insights into regulatory variation. Nat Rev Genet 2010, 11(8):533-538.

13. Li M, Wang IX, Li Y, Bruzel A, Richards AL, Toung JM, Cheung VG: Widespread RNA and DNA sequence differences in the human transcriptome. Science 2011, 333(6038):53-58.

14. Pruitt KD, Tatusova T, Klimke W, Maglott DR: NCBI Reference Sequences: current status, policy and new initiatives. Nucleic Acids Res 2009, 37(Database issue):D32-D36.

15. Langmead B, Trapnell C, Pop M, Salzberg SL: Ultrafast and memoryefficient alignment of short DNA sequences to the human genome. Genome Biol 2009, 10(3):R25.

16. Koehler R, Issac H, Cloonan N, Grimmond SM: The uniqueome: a mappability resource for short-tag sequencing. Bioinformatics 2011, 27(2):272-274.

17. Trapnell C, Roberts A, Goff L, Pertea G, Kim D, Kelley DR, Pimentel H, Salzberg SL, Rinn JL, Pachter L: Differential gene and transcript expression analysis of RNA-seq experiments with TopHat and Cufflinks. Nat Protoc 2012, 7(3):562-578

18. van de Graaf SA, Ris-Stalpers C, Pauws E, Mendive FM, Targovnik HM, de Vijlder JJ: Up to date with human thyroglobulin. J Endocrinol 2001, 170(2):307-321.

19. Kent WJ, Sugnet CW, Furey TS, Roskin KM, Pringle TH, Zahler AM, Haussler D: The human genome browser at UCSC. Genome Res 2002, 12(6):996-1006.

20. Cabili MN, Trapnell C, Goff L, Koziol M, Tazon-Vega B, Regev A, Rinn JL: Integrative annotation of human large intergenic noncoding RNAs reveals global properties and specific subclasses. Genes Dev 2011, 25(18):1915-1927.

21. Trapnell C, Pachter L, Salzberg SL: TopHat: discovering splice junctions with RNA-Seq. Bioinformatics 2009, 25(9):1105-1111.

22. Quinlan AR, Hall IM: BEDTools: a flexible suite of utilities for comparing genomic features. Bioinformatics 2010, 26(6):841-842.

23. Karro JE, Yan $Y$, Zheng D, Zhang Z, Carriero N, Cayting P, Harrrison P, Gerstein M: Pseudogene.org: a comprehensive database and comparison platform for pseudogene annotation. Nucleic Acids Res 2007, 35(Database issue):D55-D60.

doi:10.1186/1471-2164-13-412

Cite this article as: Tonner et al.: Detecting transcription of ribosomal protein pseudogenes in diverse human tissues from RNA-seq data. BMC Genomics 2012 13:412. 